\title{
Preparation of Multifunctional Metal Oxynitride 2D Crystals and Oriented Transparent Free-Standing Oxynitride Films
}

\author{
Chu-Wei Hsu, ${ }^{1}$ Takumi Ideta, ${ }^{2}$ Keisuke Awaya, ${ }^{1}$ Masayuki Tsushida, ${ }^{2}$ Tetsuya Sato, ${ }^{2}$ Kei-ichi Yanagisawa, ${ }^{3}$ Koji Kimoto, ${ }^{3}$ Kazuto \\ Hatakeyama, ${ }^{1}$ Michio Koinuma, ${ }^{1,2}$ Shintaro Ida ${ }^{1,2 *}$ \\ ${ }^{1}$ Institute of Industrial Nanomaterials, Kumamoto University, 2-39-1 Kurokami, Chuo-ku, Kumamoto 860-8555, Japan \\ ${ }^{2}$ Graduate School of Science and Technology, Kumamoto University, 2-39-1 Kurokami Chuo-ku, Kumamoto 860-8555, Japan. \\ ${ }^{3}$ Research Center for Advanced Measurement and Characterization, National Institute for Materials Science, 1-2-1 Sengen, \\ Tsukuba, Ibaraki 305-0044, Japan
}

Corresponding author

ida-s@kumamoto-u.ac.jp (Shintaro Ida)

\section{Table of Contents}

Figure S1. (a) Spectral distribution of the 300W xenon lamp (MAX302, Asahi Spectra Co., Ltd), which is used as the UV-light source in this study, (b) the layout drawing for the test cell and irradiation area.

Figure S2. The color of sample after nitridation treatment of $\mathrm{NaCa}_{2} \mathrm{Ta}_{3} \mathrm{O}_{10}$ with $\mathrm{Na}_{2} \mathrm{CO}_{3}$ or $\mathrm{NaCl}$ at $850{ }^{\circ} \mathrm{C}$.

Figure S3. XRD patters of layered oxynitride after nitridation treatment at different $\mathrm{NH}_{3}$ flow rate.

Figure S4. XRD patters of layered oxynitride after nitridation treatment at different temperature.

Figure S5. XRD patters of layered oxynitride after nitridation treatment at different reaction time.

Figure S6. XRD patters of layered oxynitride after nitridation treatment at different $\mathrm{NaCa}_{2} \mathrm{Ta}_{3} \mathrm{O}_{10} / \mathrm{NaCl}$ ratios.

Figure S7. Rietveld refinement patterns of the $\mathrm{Na}_{1.7} \mathrm{Ca}_{2} \mathrm{Ta}_{3} \mathrm{O}_{9.3} \mathrm{~N}_{0.7}$.

Figure S8. XRD pattern of $\mathrm{Na}_{1.7} \mathrm{Ca}_{2} \mathrm{Ta}_{3} \mathrm{O}_{9.3} \mathrm{~N}_{0.7}$, the protonated form, and EA-intercalated form.

Figure S9. Out-of-plane ( $2 \theta / \theta$ scan) XRD patterns of narrow scan on the 001,002 , and 003 reflections over (a) oxynitride 2D-[Ca2 $\left.\mathrm{Ta}_{3} \mathrm{O}_{9.5} \mathrm{~N}_{0.5}\right]^{-1.5}$ nanosheet deposited on Si wafer, and (b) layered oxynitride $\mathrm{Na}_{2} \mathrm{Ca}_{2} \mathrm{Ta}_{3} \mathrm{O}_{9} \mathrm{~N}$.

Figure S10. Survey XPS spectra of layered oxynitride and the protonated form.

Figure S11. XPS spectra of Ta4f, Ta4p, and N1s for layered oxynitride and the protonated form.

Figure S12. XPS spectra of $\mathrm{Na} 1 \mathrm{~s}$ and $\mathrm{Ca} 2 \mathrm{p}$ for layered oxynitride and the protonated form.

Figure S13. XPS spectra of $O 1 \mathrm{~s}$ and $\mathrm{C} 1 \mathrm{~s}$ for layered oxynitride and the protonated form.

Figure S14. TG and DTA profiles of $\mathrm{Na}_{1.7} \mathrm{Ca}_{2} \mathrm{Ta}_{3} \mathrm{O}_{9.3} \mathrm{~N}_{0.7}$.

Figure S15. TPD profiles of $\mathrm{Na}_{1.7} \mathrm{Ca}_{2} \mathrm{Ta}_{3} \mathrm{O}_{9.3} \mathrm{~N}_{0.7}\left(\mathrm{O}_{2} / \mathrm{Ar}=2: 8\right.$ flow $)$.

Figure S16. Total density of state (DOS), some partial DOSs, and the structural model of $\mathrm{Na}_{1.5} \mathrm{Ca}_{2} \mathrm{Ta}_{3} \mathrm{O}_{9.5} \mathrm{~N}_{0.5}$.

Figure S17. Bandgap of $\mathrm{Na}_{2} \mathrm{Ca}_{2} \mathrm{Ta}_{3} \mathrm{O}_{9} \mathrm{~N}$ with different $\mathrm{N}$-doped sites.

Figure S18. In-plane phi-scan XRD pattern of the monolayer $\mathrm{Ca}_{2} \mathrm{Ta}_{3} \mathrm{O}_{9.5} \mathrm{~N}_{0.5} 2 \mathrm{D}$ crystal film.

Figure S19. Cross sectional SEM image of hydrogen fuel cell using $\mathrm{Ca}_{2} \mathrm{Ta}_{3} \mathrm{O}_{9} \mathrm{~N}$ film as an electrolyte.

Figure S20. Time courses of hydrogen and oxygen generation over $\mathrm{Na}^{+}$restacked $\mathrm{Ca}_{2} \mathrm{Ta}_{3} \mathrm{O}_{9.5} \mathrm{~N}_{0.5} 2 \mathrm{D}$ crystal (Cocatalyst: $0.5 \mathrm{wt} \% \mathrm{RhCrO}_{x}$ and $0.5 \mathrm{wt} \% \mathrm{CoO}_{x}$ ). Reaction conditions: catalyst, $20 \mathrm{mg}$ dissolved in $20 \mathrm{~mL}$ of distilled water; light source, LED visible light $(\lambda=420 \mathrm{~nm})$.

Table S1. The light intensity and illumination area of monochromatic wavelength UV-light and LED light source. 
(a)

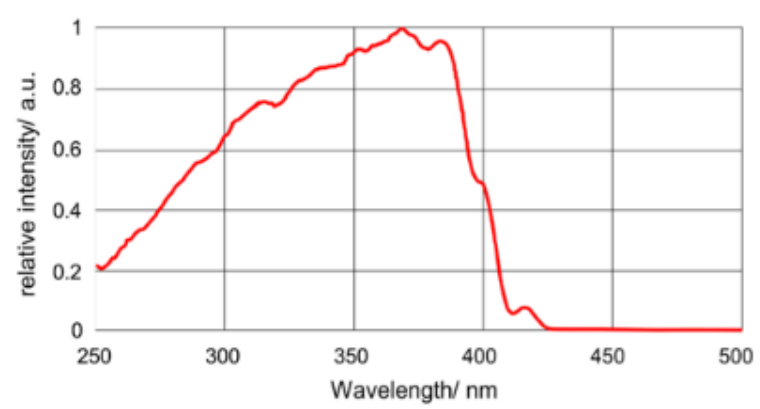

(b)

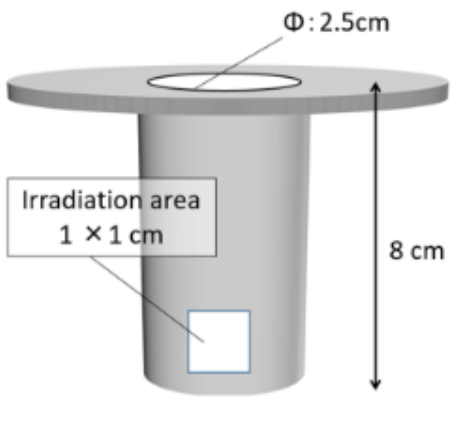

Figure S1. (a) Spectral distribution of the 300W xenon lamp (MAX302, Asahi Spectra Co., Ltd), which is used as the UV-light source in this study, (b) the layout drawing for the test cell and irradiation area. 

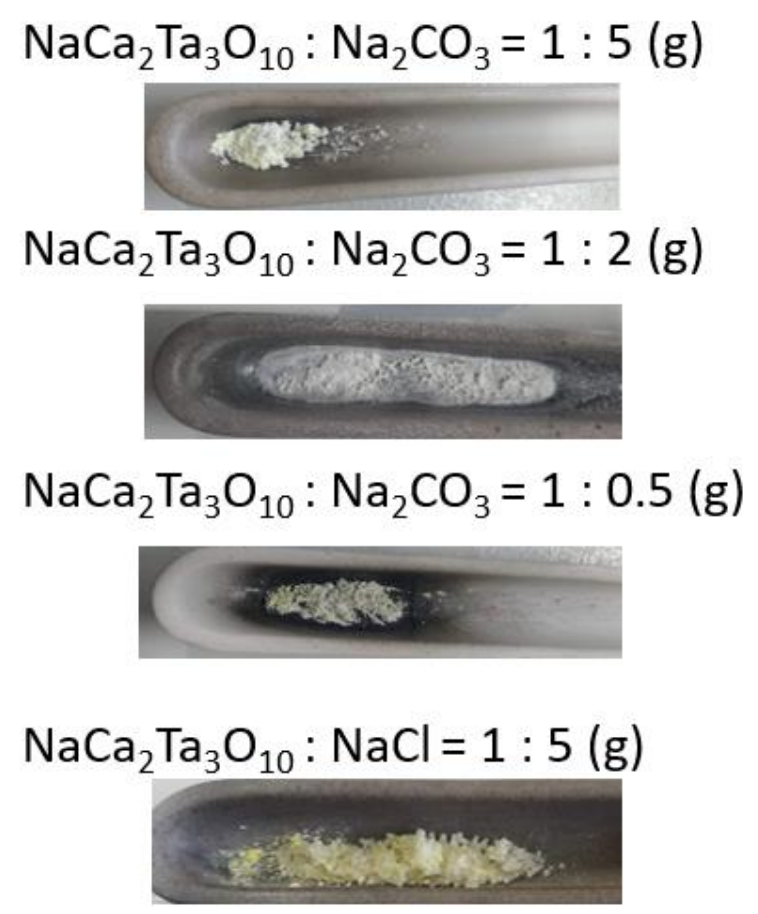

$\mathrm{NaCa}_{2} \mathrm{Ta}_{3} \mathrm{O}_{10}: \mathrm{NaCl}=1: 2$ (g)

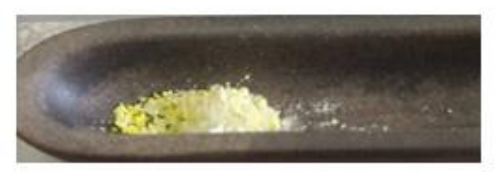

Figure S2. The color of sample after nitridation treatment of $\mathrm{NaCa}_{2} \mathrm{Ta}_{3} \mathrm{O}_{10}$ with charge compensation reagent $\left(\mathrm{Na}_{2} \mathrm{CO}_{3}\right.$ or $\left.\mathrm{NaCl}\right)$ at $850{ }^{\circ} \mathrm{C}$. In the nitriding process, $\mathrm{NaCa}_{2} \mathrm{Ta}_{3} \mathrm{O}_{10}$ is converted to $\mathrm{Na}_{2} \mathrm{Ca}_{2} \mathrm{Ta}_{3} \mathrm{O}_{9} \mathrm{~N}$, so a charge compensation reagent is required. The color of the sample prepared with $\mathrm{Na}_{2} \mathrm{CO}_{3}$ was white, but the color of the boat (crucible) became black. On the other hand, when $\mathrm{NaCl}$ was used as a charge compensation reagent, yellow samples were obtained. Therefore, in this study, $\mathrm{NaCl}$ was used as a charge compensation reagent. 


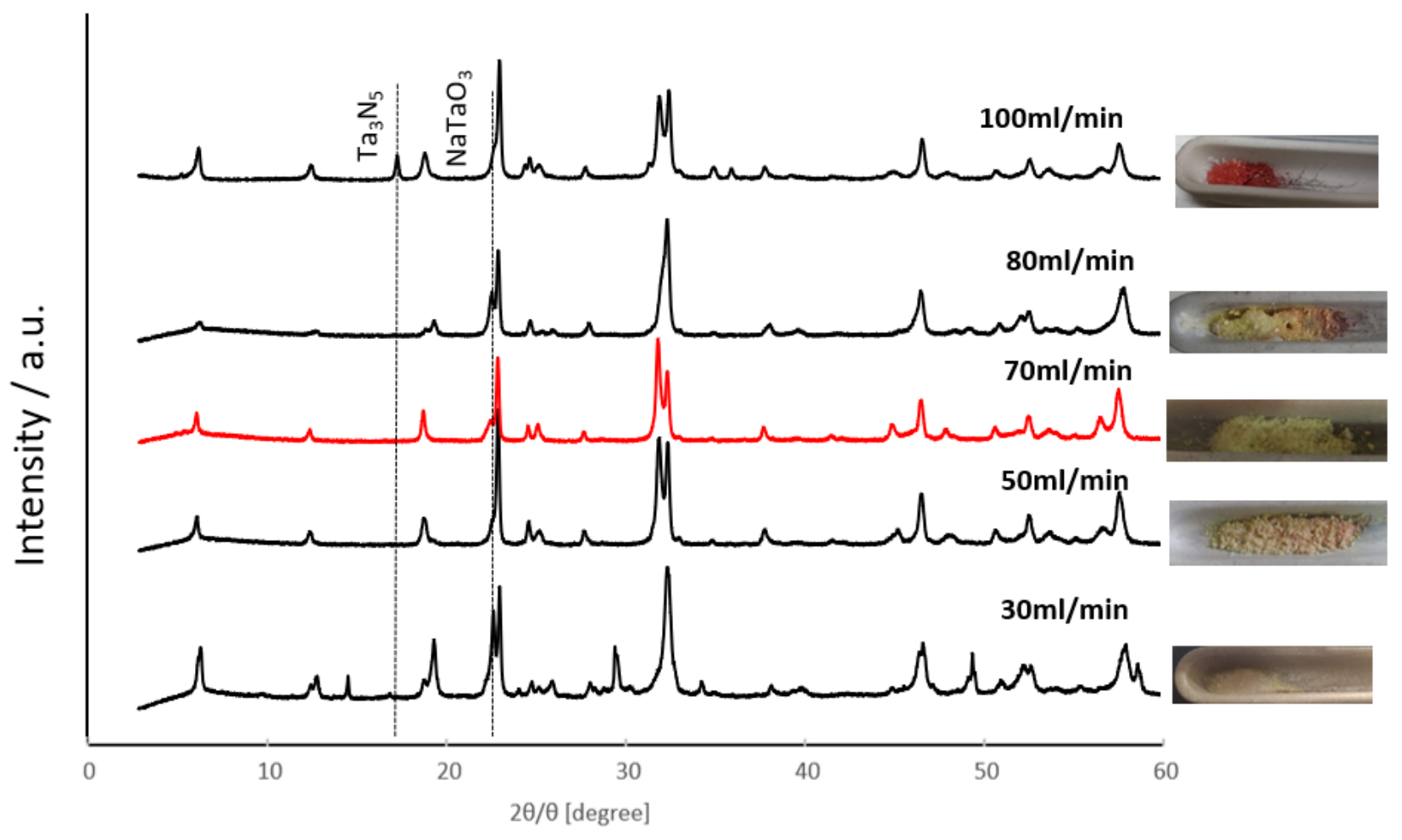

Figure S3. XRD patters of layered oxynitride after nitridation treatment at different $\mathrm{NH}_{3}$ flow rate (reaction time: $6 \mathrm{~h}, \mathrm{NH}_{3}$ reaction temperature: $850{ }^{\circ} \mathrm{C}, \mathrm{NaCa}_{2} \mathrm{Ta}_{3} \mathrm{O}_{10}: \mathrm{NaCl}=1: 5$ (g)). Although impurity phases such as $\mathrm{NaTaO}_{3}$ and $\mathrm{Ta}_{3} \mathrm{~N}_{5}$ were observed, the sample prepared at a flow rate of $70 \mathrm{ml} / \mathrm{min}$ showed low diffraction peaks assigned to the impurity phases. 


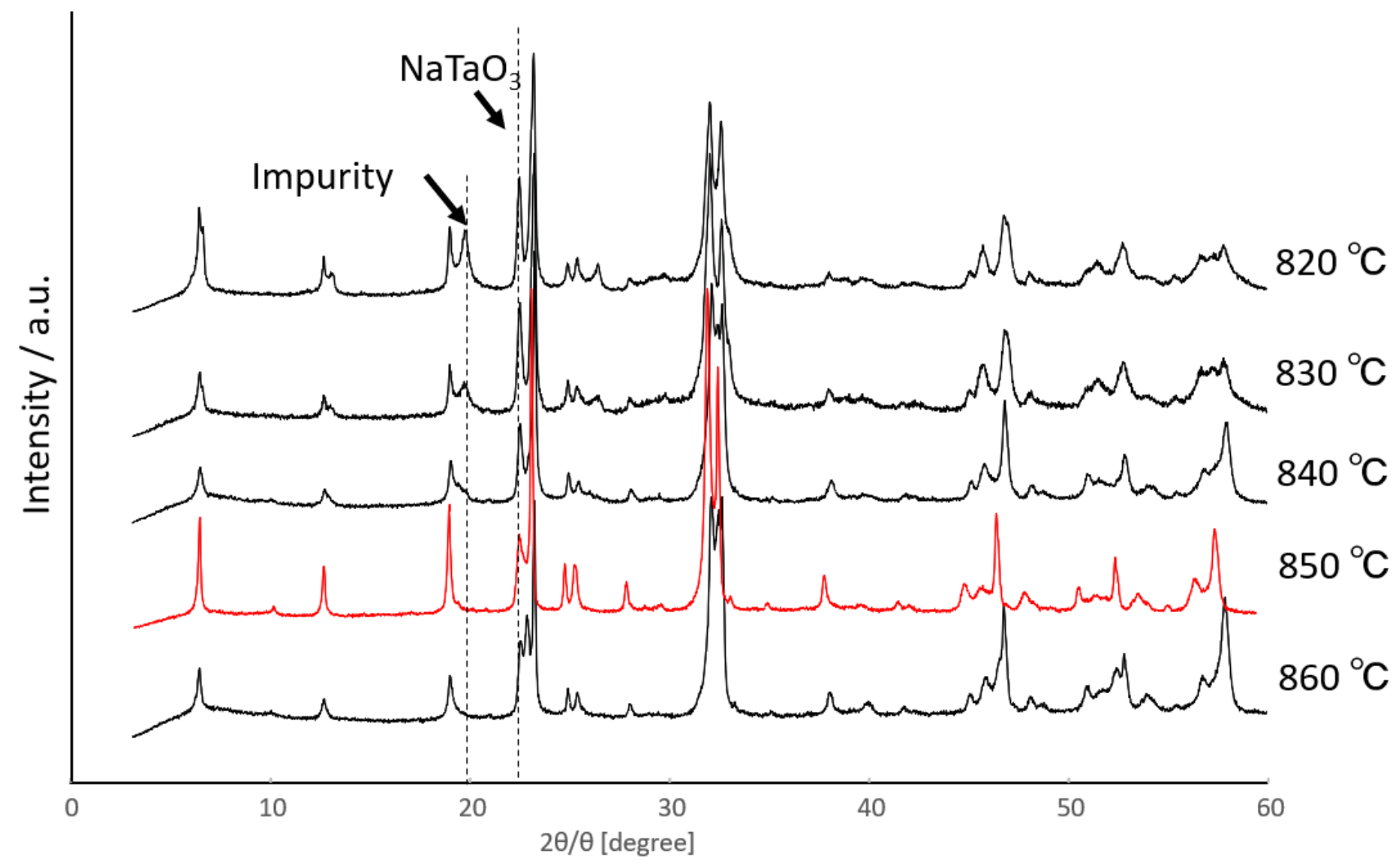

Figure S4. XRD patters of layered oxynitride after nitridation treatment at different temperature (reaction time: $2 \mathrm{~h}, \mathrm{NH}_{3}$ flow rate:70 $\mathrm{mL} / \mathrm{min}, \mathrm{NaCa}_{2} \mathrm{Ta}_{3} \mathrm{O}_{10}: \mathrm{NaCl}=1: 5(\mathrm{~g})$ ). Although impurity phases such as $\mathrm{NaTaO}_{3}$ were observed, the sample prepared at $850{ }^{\circ} \mathrm{C}$ showed low diffraction peaks assigned to the impurity phases. 


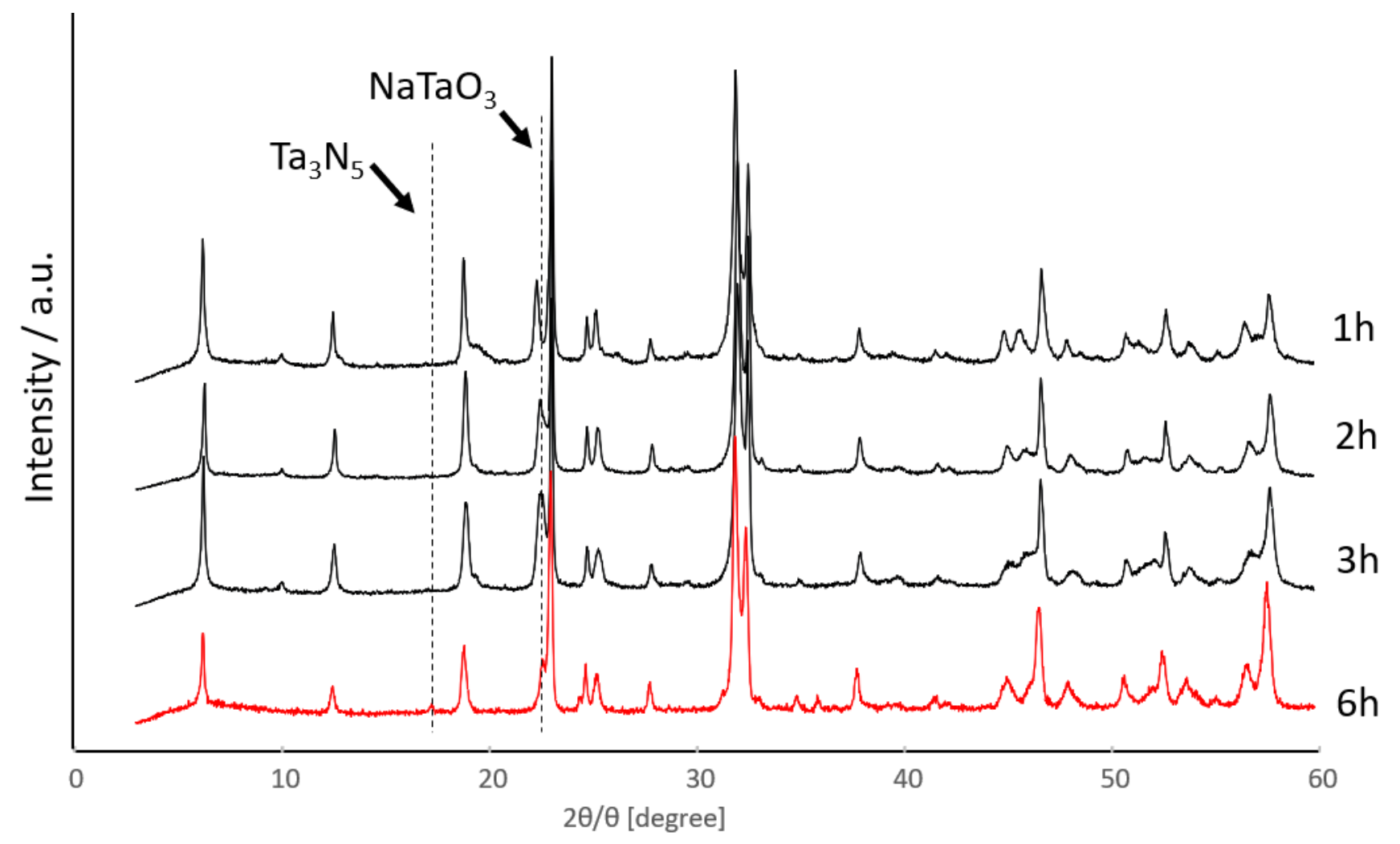

Figure S5. XRD patters of layered oxynitride after nitridation treatment at different reaction time (reaction temperature: $850{ }^{\circ} \mathrm{C}, \mathrm{NH}_{3}$ flow rate: $70 \mathrm{~mL} / \mathrm{min}, \mathrm{NaCa}_{2} \mathrm{Ta}_{3} \mathrm{O}_{10}: \mathrm{NaCl}=1: 5(\mathrm{~g})$ ). Although impurity phases such as $\mathrm{NaTaO}_{3}$ were observed, the sample prepared for $6 \mathrm{~h}$ showed low diffraction peaks assigned to the impurity phases. 


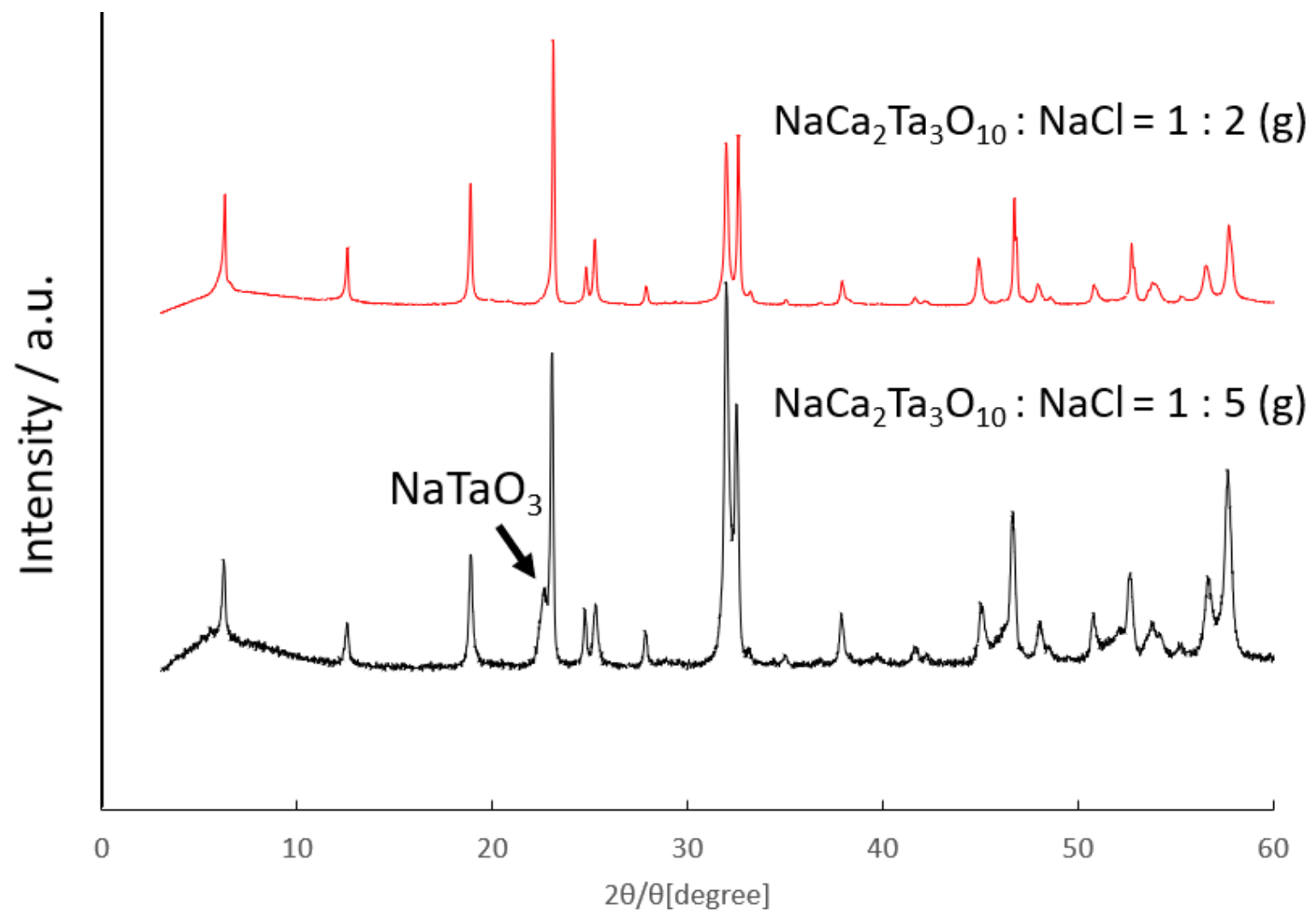

Figure S6. XRD patters of layered oxynitride after nitridation treatment at different $\mathrm{NaCa}_{2} \mathrm{Ta}_{3} \mathrm{O}_{10} / \mathrm{NaCl}$ ratios (reaction time: 6 h, reaction temperature: $850{ }^{\circ} \mathrm{C}, \mathrm{NH}_{3}$ flow rate:70 $\mathrm{mL} / \mathrm{min}$ ). The sample prepared with a $\mathrm{NaCa}_{2} \mathrm{Ta}_{3} \mathrm{O}_{10} / \mathrm{NaCl}$ ratio of $1 / 2$ showed no impurity phase. 


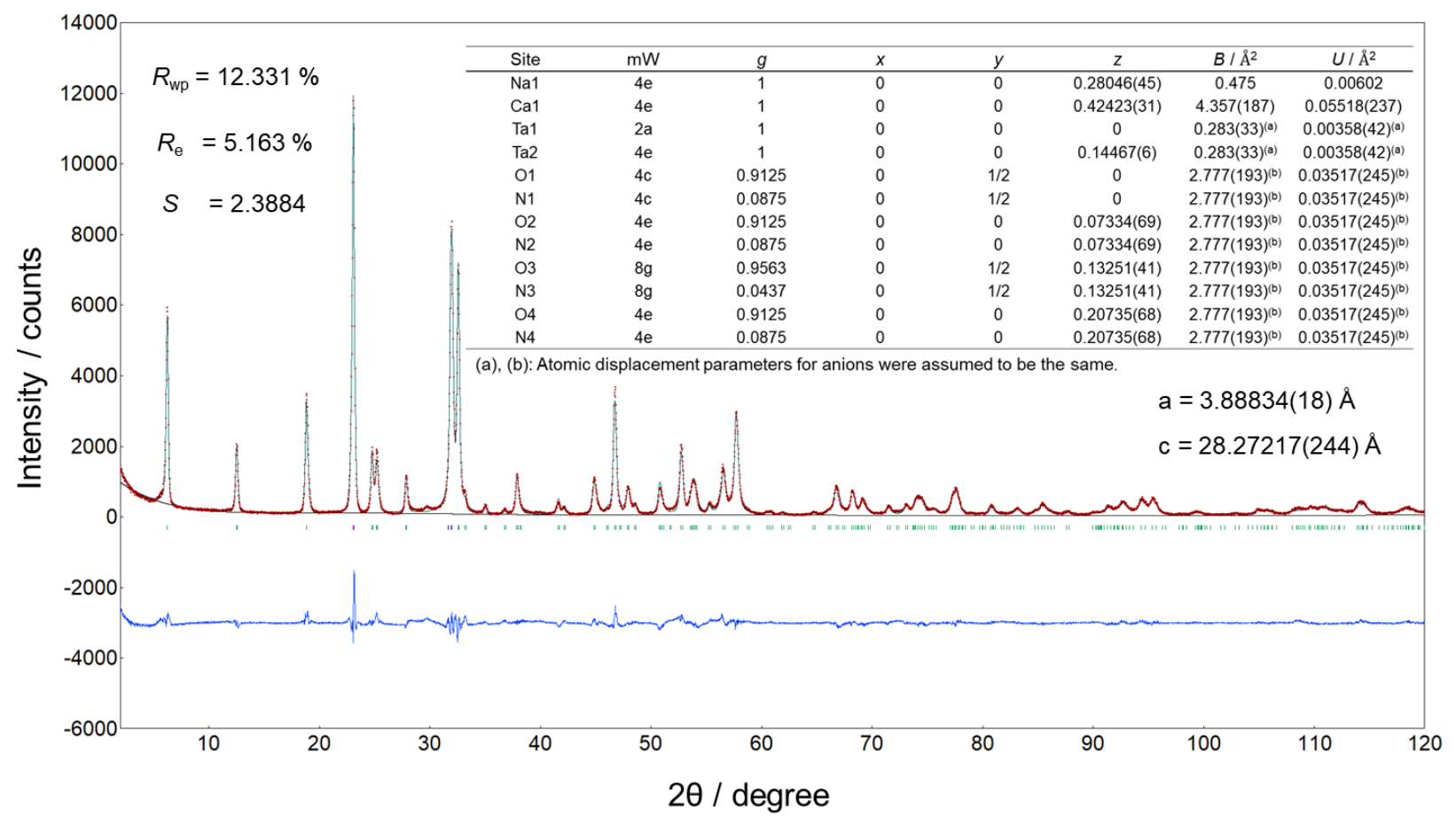

Figure S7. Rietveld refinement patterns of the $\mathrm{Na}_{1.7} \mathrm{Ca}_{2} \mathrm{Ta}_{3} \mathrm{O}_{9.3} \mathrm{~N}_{0.7}$. The final Rietveld refinement gave $R_{\mathrm{wp}}=12.3 \%, R_{\mathrm{e}}=5.2 \%$, and $S=2.9$. The Rietveld analysis was performed using RIETAN-FP. 


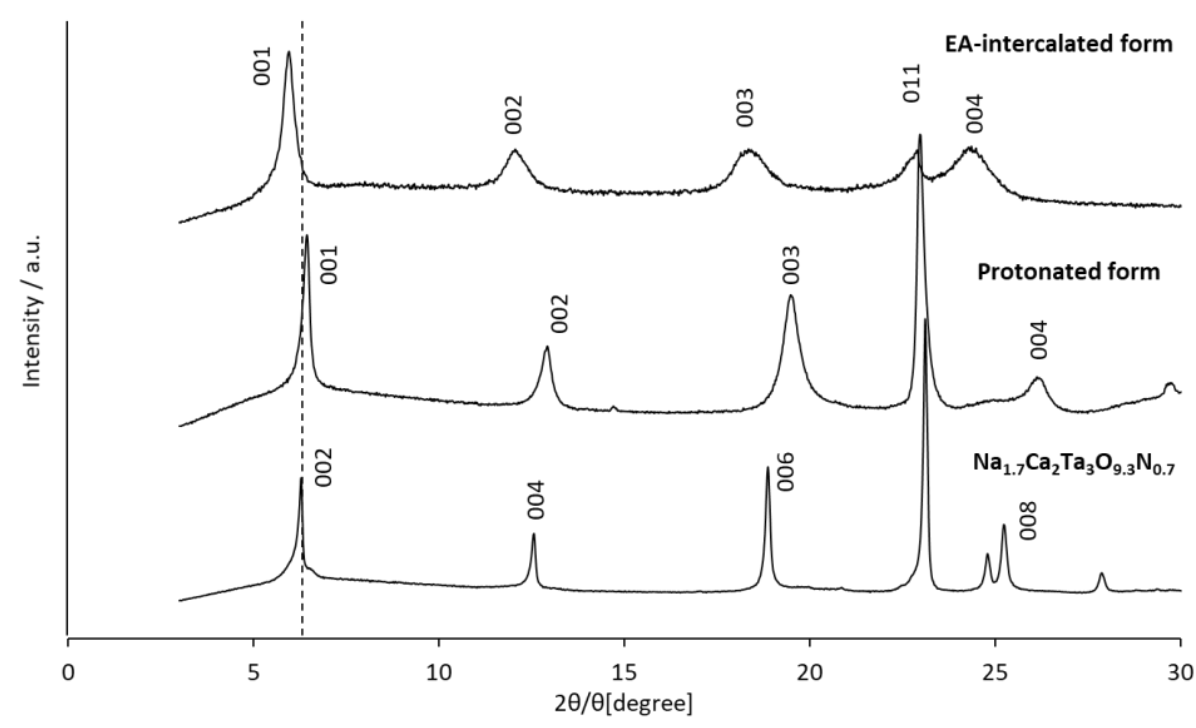

Figure S8. XRD pattern of $\mathrm{Na}_{1.7} \mathrm{Ca}_{2} \mathrm{Ta}_{3} \mathrm{O}_{9.3} \mathrm{~N}_{0.7}$, the protonated form, and EA-intercalated form. After EA intercalation, the 001 reflection peak shifted to lower angle, indicating that EA is intercalated into the interlayer of the layered compound. 


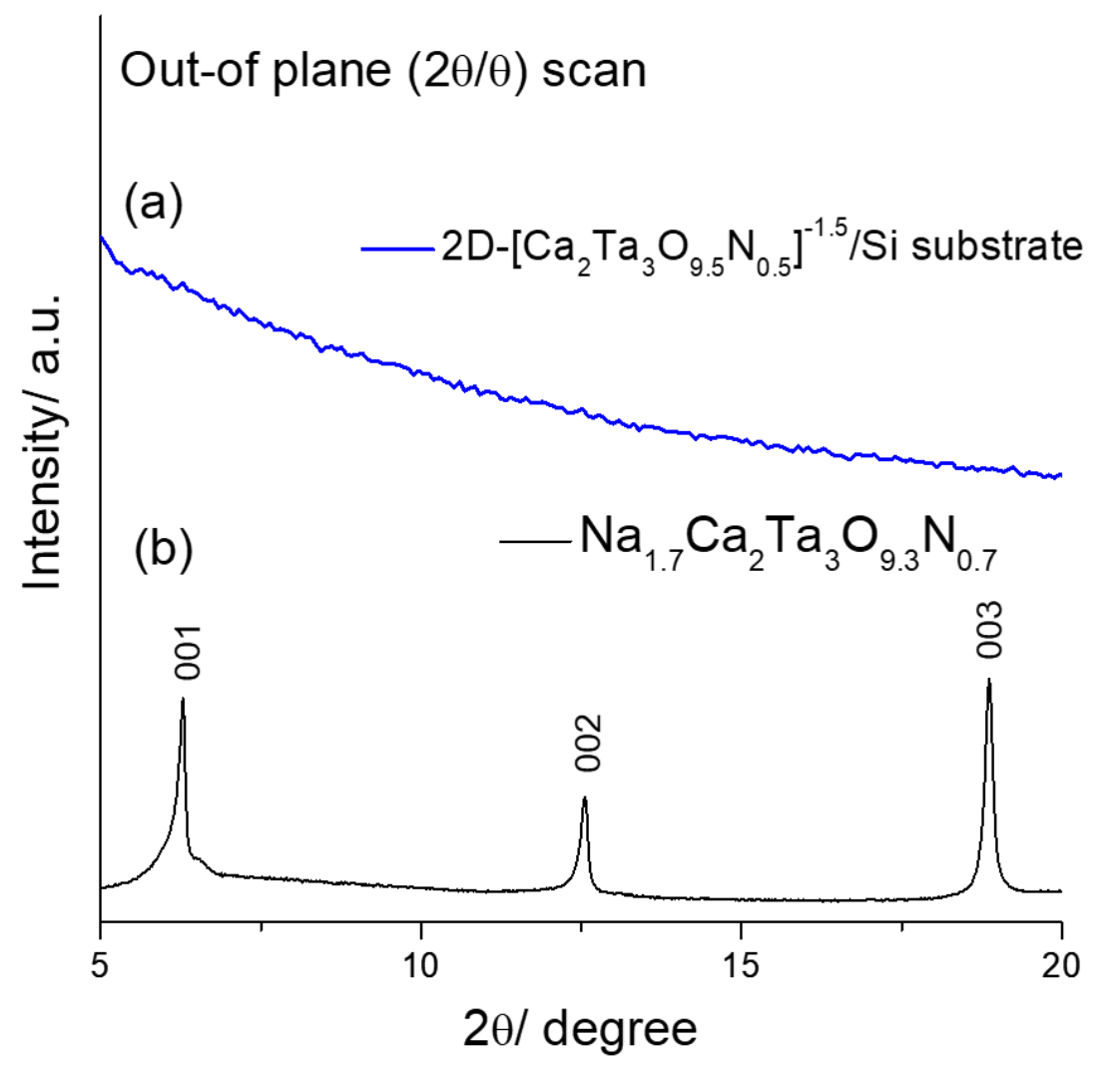

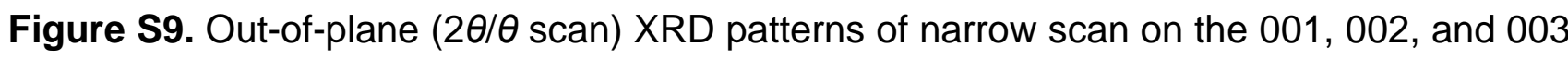
reflections over (a) oxynitride 2D-[Ca2 $\left.\mathrm{Ta}_{3} \mathrm{O}_{9.5} \mathrm{~N}_{0.5}\right]^{-1.5}$ nanosheet deposited on $\mathrm{Si}$ wafer, and (b) layered oxynitride $\mathrm{Na}_{1.7} \mathrm{Ca}_{2} \mathrm{Ta}_{3} \mathrm{O}_{9.3} \mathrm{~N}_{0.7}$. 


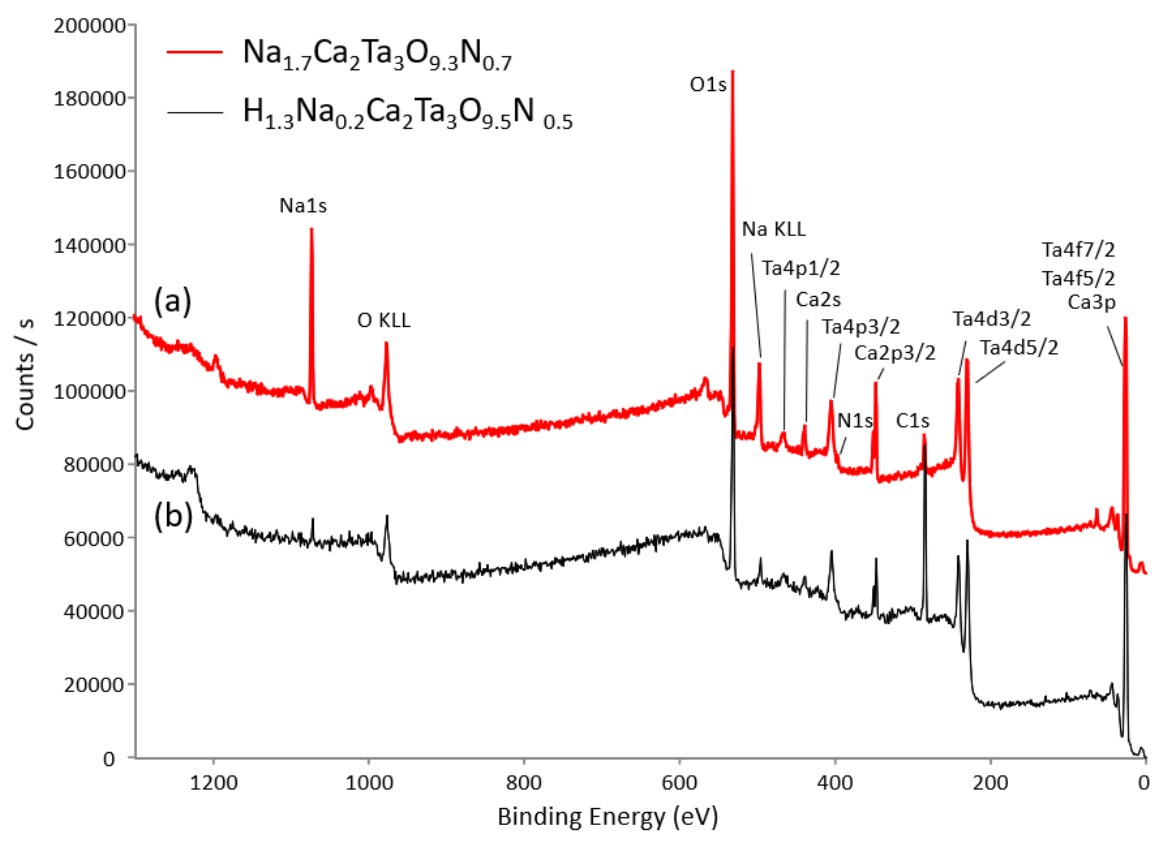

Figure S10. Survey XPS spectra of layered oxynitride $\left(\mathrm{Na}_{1.7} \mathrm{Ca}_{2} \mathrm{Ta}_{3} \mathrm{O}_{9.3} \mathrm{~N}_{0.7}\right)$ and the protonated form $\left(\mathrm{H}_{1.3} \mathrm{Na}_{0.2} \mathrm{Ca}_{2} \mathrm{Ta}_{3} \mathrm{O}_{9.5} \mathrm{~N}_{0.5}\right)$. 

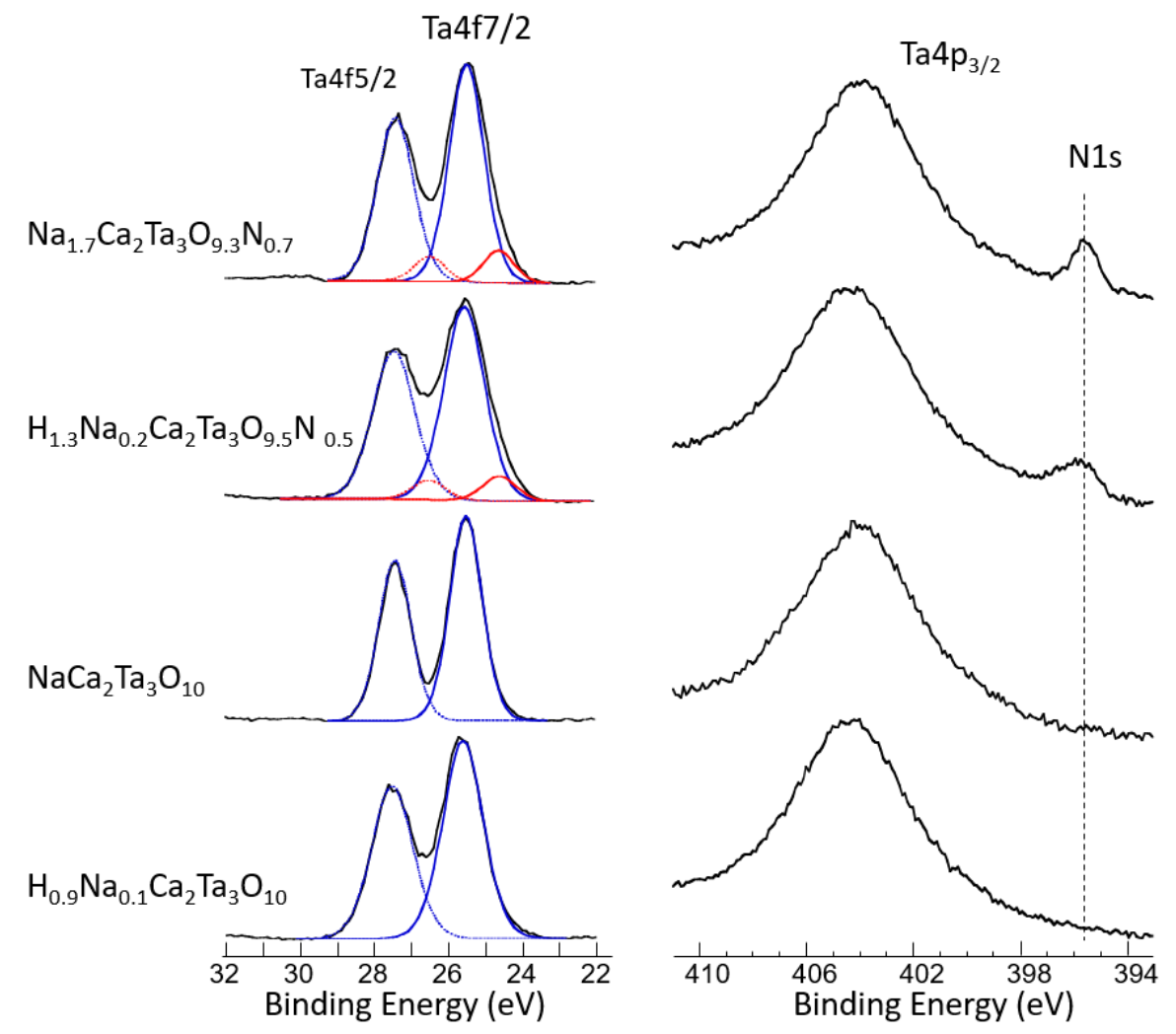

Figure S11. XPS spectra of Ta4f, Ta4p, and N1s for layered oxynitride $\left(\mathrm{Na}_{1.7} \mathrm{Ca}_{2} \mathrm{Ta}_{3} \mathrm{O}_{9.3} \mathrm{~N}_{0.7}\right)$ the protonated form $\left(\mathrm{H}_{1.3} \mathrm{Na}_{0.2} \mathrm{Ca}_{2} \mathrm{Ta}_{3} \mathrm{O}_{9.5} \mathrm{~N}_{0.5}\right)$, $\mathrm{NaCa} \mathrm{Ta}_{3} \mathrm{O}_{10}$, and the protonated form of $\mathrm{NaCa}_{2} \mathrm{Ta}_{3} \mathrm{O}_{10}\left(\mathrm{H}_{0.9} \mathrm{Na}_{0.1} \mathrm{Ca}_{2} \mathrm{Ta}_{3} \mathrm{O}_{10}\right)$. The $\mathrm{N} 1 s$ peaks were observed from $\mathrm{Na}_{1.7} \mathrm{Ca}_{2} \mathrm{Ta}_{3} \mathrm{O}_{9.3} \mathrm{~N}_{0.7}$ and $\mathrm{H}_{1.3} \mathrm{Na}_{0.2} \mathrm{Ca}_{2} \mathrm{Ta}_{3} \mathrm{O}_{9.5} \mathrm{~N}_{0.5}$. In the cases of $\mathrm{Na}_{1.7} \mathrm{Ca}_{2} \mathrm{Ta}_{3} \mathrm{O}_{9.3} \mathrm{~N}_{0.7}$ and $\mathrm{H}_{1.3} \mathrm{Na}_{0.2} \mathrm{Ca}_{2} \mathrm{Ta}_{3} \mathrm{O}_{9.5} \mathrm{~N}_{0.5}$, two types of chemical state of Ta were observed in the Ta $4 \mathrm{f}$ XPS spectra, indicating that $\mathrm{Ta}-\mathrm{N}$ and $\mathrm{Ta}-\mathrm{O}$ bondings exist in the $\mathrm{Na}_{1.7} \mathrm{Ca}_{2} \mathrm{Ta}_{3} \mathrm{O}_{9.3} \mathrm{~N}_{0.7}$ and $\mathrm{H}_{1.3} \mathrm{Na}_{0.2} \mathrm{Ca}_{2} \mathrm{Ta}_{3} \mathrm{O}_{9.5} \mathrm{~N}_{0.5}$. 

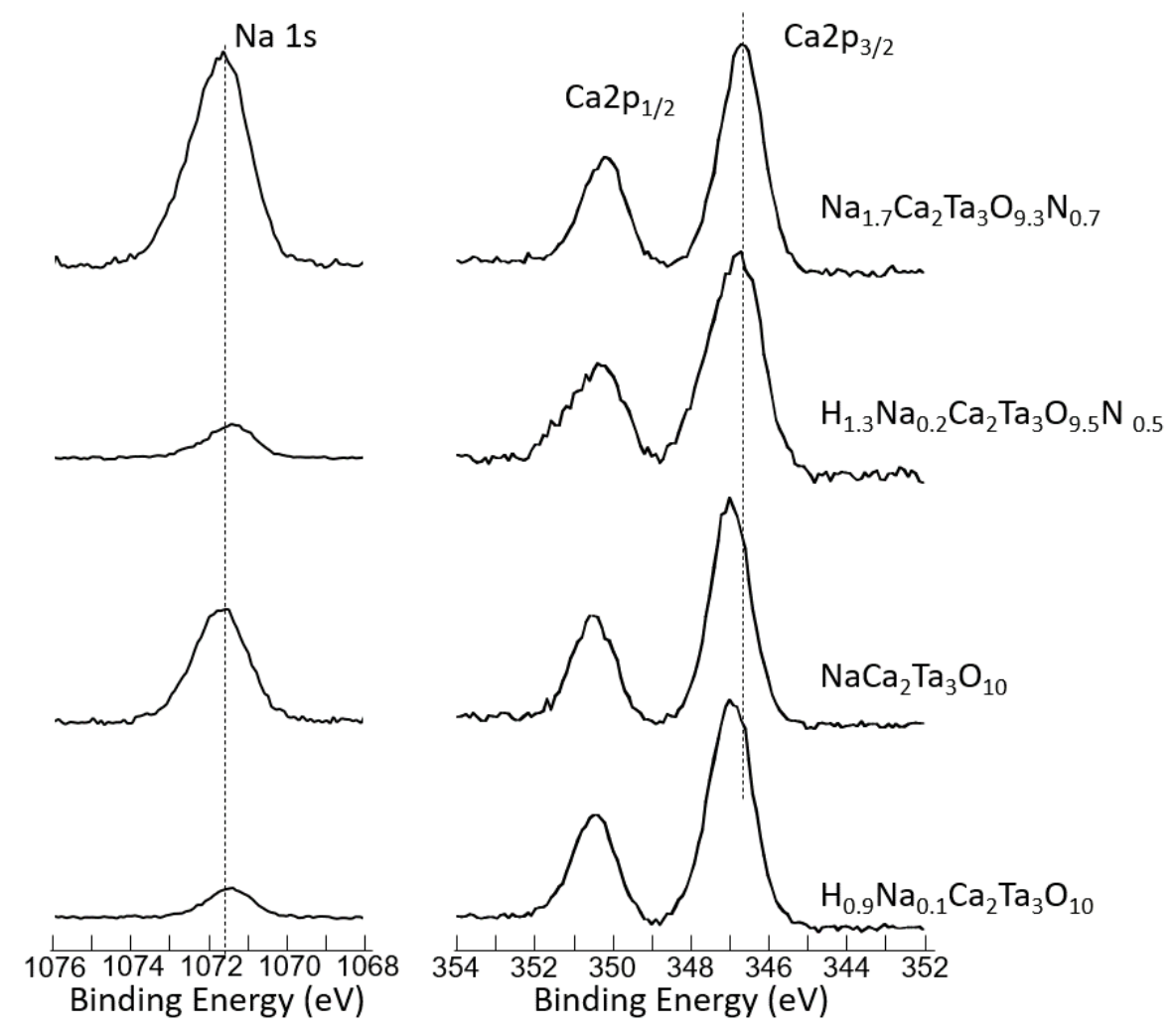

Figure S12. XPS spectra of $\mathrm{Na} 1 \mathrm{~s}$ and $\mathrm{Ca} 2 \mathrm{p}$ for layered oxynitride $\left(\mathrm{Na}_{1.7} \mathrm{Ca}_{2} \mathrm{Ta}_{3} \mathrm{O}_{9.3} \mathrm{~N}_{0.7}\right)$ the protonated form $\left(\mathrm{H}_{1.3} \mathrm{Na}_{0.2} \mathrm{Ca}_{2} \mathrm{Ta}_{3} \mathrm{O}_{9.5} \mathrm{~N}_{0.5}\right), \mathrm{NaCa}_{2} \mathrm{Ta}_{3} \mathrm{O}_{10}$, and the protonated form of $\mathrm{NaCa}_{2} \mathrm{Ta}_{3} \mathrm{O}_{10}\left(\mathrm{H}_{0.9} \mathrm{Na}_{0.1} \mathrm{Ca}_{2} \mathrm{Ta}_{3} \mathrm{O}_{10}\right)$. The signals for $\mathrm{Na} 1 \mathrm{~s}$ were decreased by acid treatment, and the peak position of $\mathrm{Ca} 2 \mathrm{p}$ in the oxynitride shifted to a slightly lower energy compared to the layered oxide. 


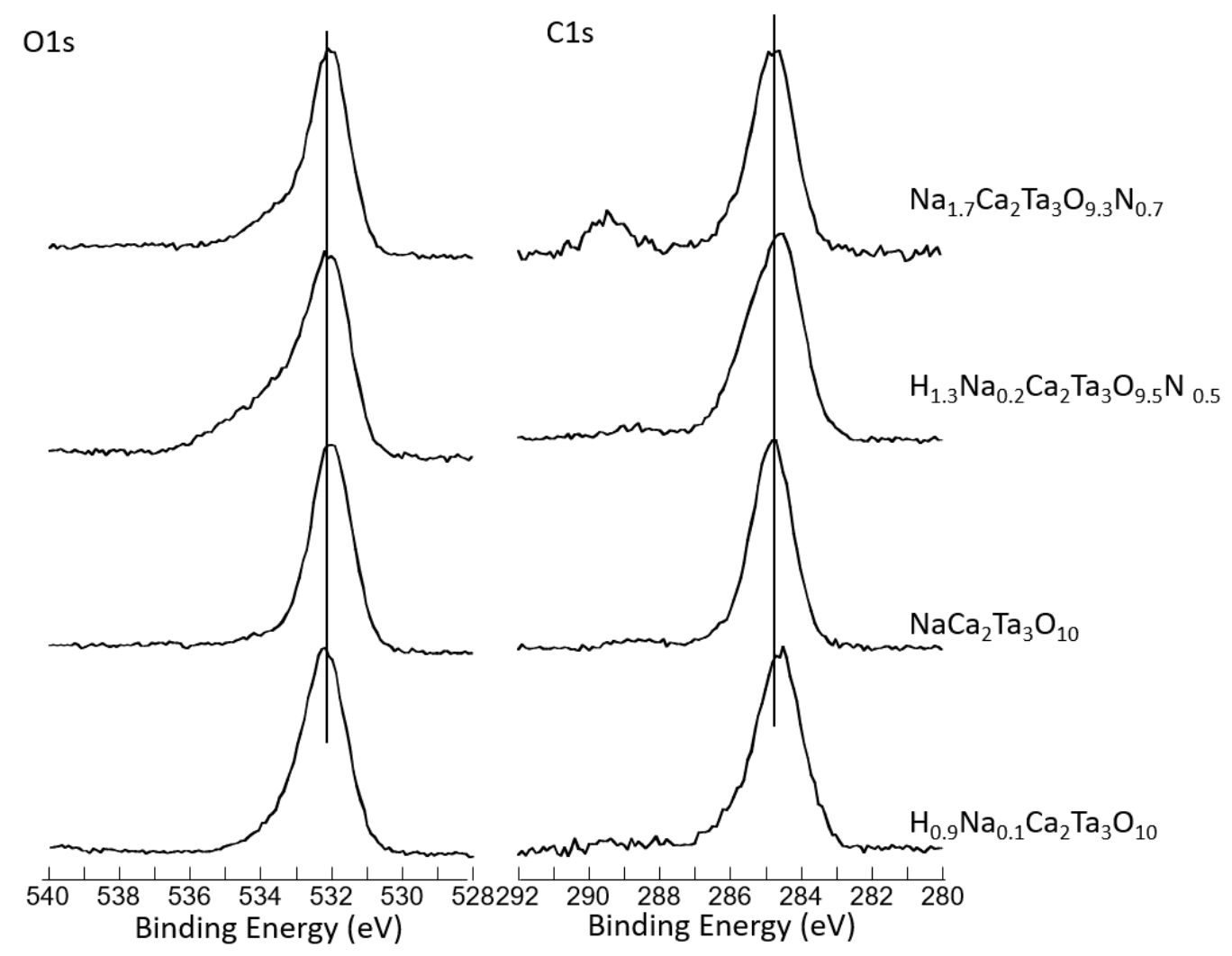

Figure S13. XPS spectra of $\mathrm{O} 1 \mathrm{~s}$ and $\mathrm{C} 1 \mathrm{~s}$ for layered oxynitride $\left(\mathrm{Na}_{1.7} \mathrm{Ca}_{2} \mathrm{Ta}_{3} \mathrm{O}_{9.3} \mathrm{~N}_{0.7}\right)$ the protonated form $\left(\mathrm{H}_{1.3} \mathrm{Na}_{0.2} \mathrm{Ca}_{2} \mathrm{Ta}_{3} \mathrm{O}_{9.5} \mathrm{~N}_{0.5}\right)$, $\mathrm{NaCa}_{2} \mathrm{Ta}_{3} \mathrm{O}_{10}$, and the protonated form of $\mathrm{NaCa}_{2} \mathrm{Ta}_{3} \mathrm{O}_{10}\left(\mathrm{H}_{0.9} \mathrm{Na}_{0.1} \mathrm{Ca}_{2} \mathrm{Ta}_{3} \mathrm{O}_{10}\right)$. The $\mathrm{O}$ 1s spectra shows that the oxynitride samples have several types of oxygen states. 


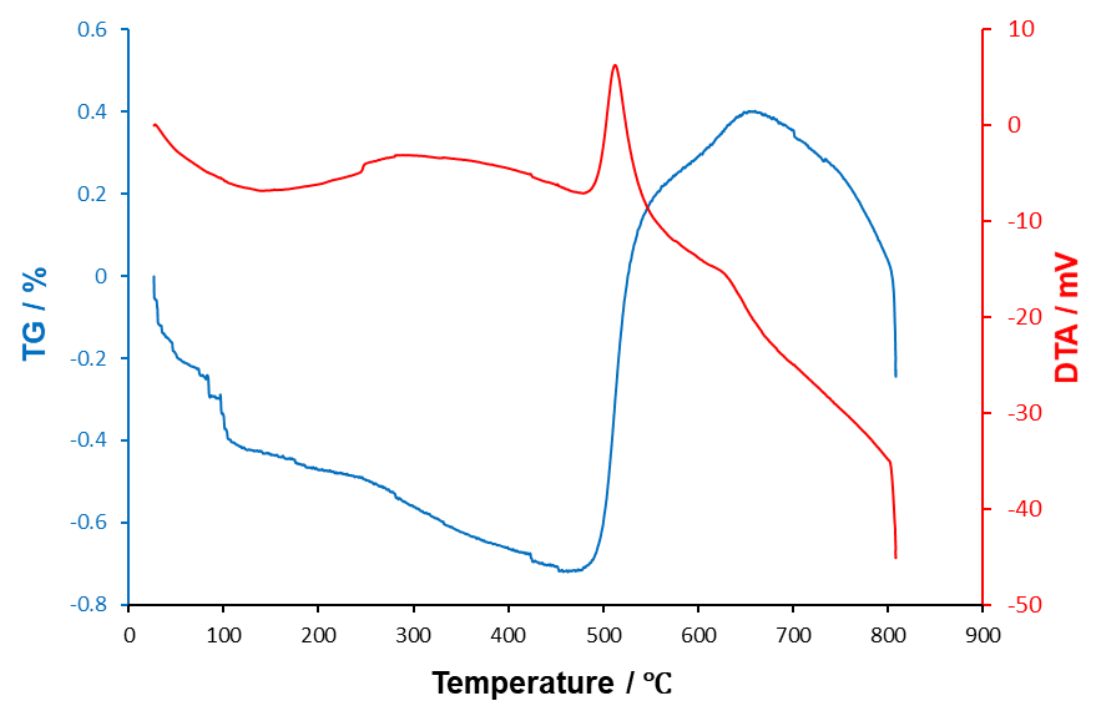

Figure S14. TG and DTA profiles of $\mathrm{Na}_{1.7} \mathrm{Ca}_{2} \mathrm{Ta}_{3} \mathrm{O}_{9.3} \mathrm{~N}_{0.7}$. From the weight loss at the initial stage $\left(<100^{\circ} \mathrm{C}\right)$, the intercalated or absorbed water in this sample was estimated as follows: $\mathrm{Na}_{1.7} \mathrm{Ca}_{2} \mathrm{Ta}_{3} \mathrm{O}_{9.3} \mathrm{~N}_{0.7} \cdot 0.15 \mathrm{H}_{2} \mathrm{O}$. The weight increase at $500-700{ }^{\circ} \mathrm{C}$ was due to replacement of nitrogen with oxygen, which was confirmed by TPD analysis (Figure S15). 


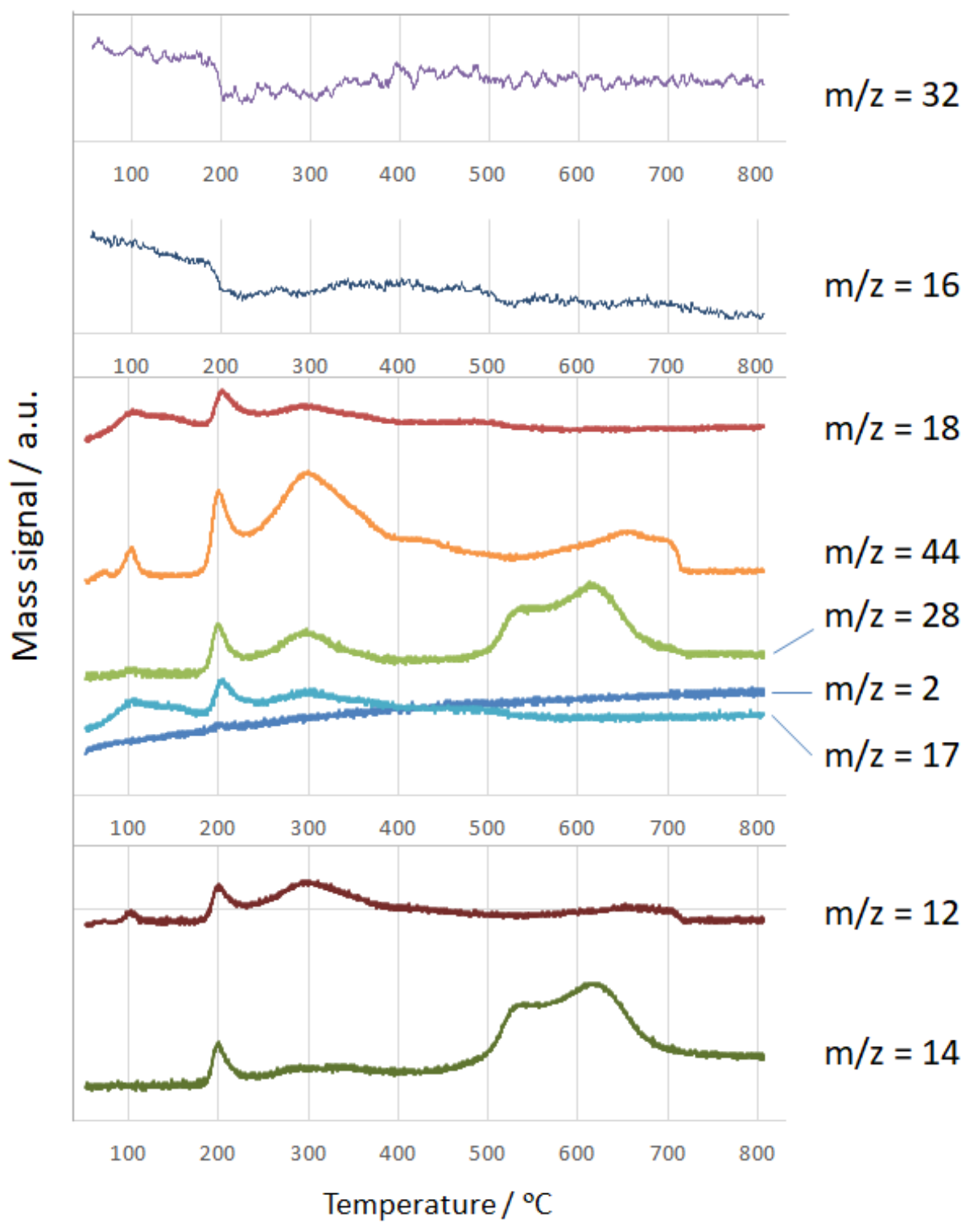

Figure S15. TPD profiles of $\mathrm{Na}_{1.7} \mathrm{Ca}_{2} \mathrm{Ta}_{3} \mathrm{O}_{9 .} \mathrm{N}_{0.7}\left(\mathrm{O}_{2} / \mathrm{Ar}=2: 8\right.$ flow). Mass signals of $\mathrm{N}_{2}(28)$ and $\mathrm{N}(14)$ were increased at $200{ }^{\circ} \mathrm{C}$ and $500-700{ }^{\circ} \mathrm{C}$. Desorption of $\mathrm{N}_{2}$ at $500-700{ }^{\circ} \mathrm{C}$ was larger than that at $200{ }^{\circ} \mathrm{C}$, indicating that nitrogen in the layered oxynitride is relatively stable under $450{ }^{\circ} \mathrm{C}$. The oxygen consumption was observed at $200{ }^{\circ} \mathrm{C}$ and $520{ }^{\circ} \mathrm{C}$. The $\mathrm{CO}_{2}$ (44) desorption was observed at $100,200,300{ }^{\circ} \mathrm{C}$, indicating that $\mathrm{CO}_{2}$ is easily absorbed on the layered oxynitride. 

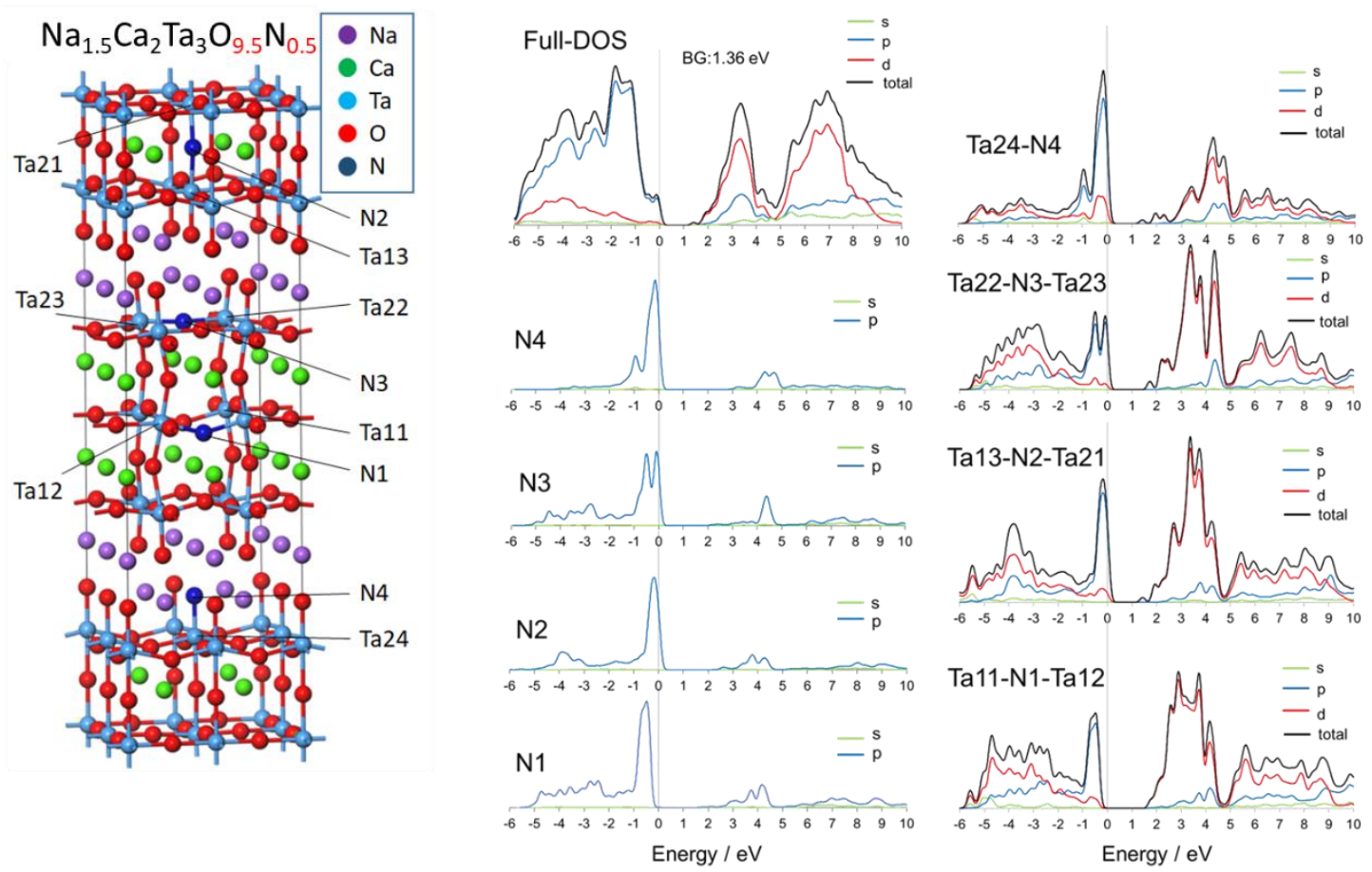

\begin{tabular}{|c|c|c|c|c|c|c|c|c|c|c|c|c|c|c|}
\hline \multicolumn{12}{|c|}{ Lattice parameters $(\hat{A})$} & & & \\
\hline \multirow{2}{*}{\multicolumn{5}{|c|}{$\begin{array}{l}a=29.387164 \\
b=8.115136 \\
c=8.110057\end{array}$}} & \multicolumn{5}{|c|}{ Fractional coordinates of atoms } & & & & & \\
\hline & & & & & & 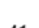 & u & 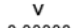 & & & & $\mathrm{u}$ & 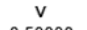 & w \\
\hline & & & & & 0 & 41 & 0,0675 & 0,0000 & 0.0835 & & 13 & 0.2845 & 0.50000 & -0.00978 \\
\hline \multirow{2}{*}{\multicolumn{5}{|c|}{ Fractional coordinates of atoms }} & & 42 & 0.20732 & 0.00000 & -0.06360 & 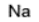 & 14 & 0.71007 & 0.50000 & 0.04922 \\
\hline & & & & & $\mathrm{o}$ & 43 & 0.93285 & 0.00000 & -0.05584 & $\mathrm{Na}$ & 15 & 0.29185 & 0.50000 & 0.54932 \\
\hline & & $\mathrm{u}$ & v & w & $\circ$ & 44 & 0.79100 & 0.00000 & 0.06755 & $\mathrm{Na}$ & 16 & 0.71199 & 0.50000 & .48446 \\
\hline $\mathrm{N}$ & 1 & 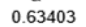 & 0.5000 & 0.28508 & & $\begin{array}{l}45 \\
46\end{array}$ & $\begin{array}{l}-0.02212 \\
0.50450\end{array}$ & $\begin{array}{l}0.00000 \\
0.00000\end{array}$ & $\begin{array}{l}0.26419 \\
0.17813\end{array}$ & $\begin{array}{l}\mathrm{Ca} \\
\mathrm{Ca}\end{array}$ & $\frac{1}{2}$ & $\begin{array}{l}0.92736 \\
0.07776\end{array}$ & $\begin{array}{l}0.25342 \\
0.26405\end{array}$ & $\begin{array}{l}0.21337 \\
0.25081\end{array}$ \\
\hline $\mathrm{N}$ & 2 & 0.21226 & 0.50000 & 0.52468 & o & 47 & 0.15443 & 0.00000 & $\begin{array}{l}0.178293 \\
0.24292\end{array}-3$ & $\begin{array}{l}\mathrm{Ca} \\
\mathrm{Ca}\end{array}$ & $\frac{2}{3}$ & 0.92355 & $\begin{array}{l}0.26455 \\
0.2456\end{array}$ & $\begin{array}{l}0.25081 \\
0.74448\end{array}$ \\
\hline $\mathrm{N}$ & 3 & 0.92907 & 0.50000 & 0.57881 & 0 & 48 & 0.62679 & 0.00000 & 0.20454 & $\mathrm{Ca}$ & 4 & 0.07322 & 0.25578 & 0.70960 \\
\hline $\mathrm{N}$ & 4 & 0.49098 & 0.50000 & 0.68092 & o & 49 & 0.88068 & 0.00000 & 0.24845 & $\mathrm{Ca}$ & 5 & 0.92736 & 0.74658 & 0.21337 \\
\hline o & 1 & 0.56601 & 0.27101 & 0.16904 & o & 50 & 0.35 & $0.0 \mathrm{c}$ & & $\mathrm{Ca}$ & 6 & & & 0.25081 \\
\hline 0 & 2 & 0.70700 & 0.22788 & 0.27821 & o & 51 & 0.06688 & 0.00 & 0.4 & $\mathrm{Ca}$ & 7 & 0.9 & 0.7 & 0.74448 \\
\hline o & 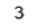 & 43132 & 0.20649 & & o & 52 & 0.21135 & 0.00000 & 0.54 & $\mathrm{Ca}$ & 8 & 0.07322 & 0.7 & 0.70960 \\
\hline o & 4 & 0.2909 & & & o & 53 & 0.93141 & 0.00000 & 0.58170 & $\mathrm{Ca}$ & 9 & 0.43644 & 0.00000 & 01733 \\
\hline o & 5 & 0.48129 & 0.282 & -0.00962 & o & 54 & 0.79319 & 0.00000 & 0.45235 & $\mathrm{Ca}$ & 10 & 0.57110 & 0.00000 & 00589 \\
\hline o & 6 & -0.00383 & 0.24968 & 0.03715 & o & 55 & 0.02243 & 0.00000 & 0.76381 & $\mathrm{Ca}$ & 11 & 0.42157 & .00000 & .49163 \\
\hline o & 7 & 0.64958 & 0.29323 & -0.00740 & o & 56 & 0.51484 & 0.00 & & a & 12 & & 0000 & 55458 \\
\hline o & 8 & 0.12785 & 0.24932 & -0.00870 & 0 & 57 & & & & $\mathrm{Ca}$ & 13 & & & \\
\hline o & 9 & 0.37673 & 0.20550 & -0.00354 & o & 58 & & & & $\mathrm{Ca}$ & 14 & 76 & 100 & 345 \\
\hline o & 10 & & & & o & 59 & 0.84582 & 0.00000 & 0.75 & $\mathrm{Ca}$ & 15 & 0.42295 & 0.50000 & 0.50194 \\
\hline & 11 & & & & 0 & 60 & 0.35466 & 0.00000 & 0.69001 & $\mathrm{Ca}$ & 16 & 0.57854 & 0.50000 & 0.54050 \\
\hline 0 & 12 & 0.71199 & 0.21670 & 0.697 & o & 61 & 0.06663 & 0.50000 & 0.07515 & $\mathrm{Ta}$ & 1 & 0.64128 & 0.25301 & 0.25906 \\
\hline o & 13 & 43258 & 0.20650 & 0.687 & o & 62 & & & & 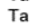 & 2 & & & \\
\hline o & 14 & 294 & 0.28178 & 0.78229 & o & 63 & & & & a & 3 & & & \\
\hline o & 15 & 0.51684 & 0.20516 & 0.48682 & o & 64 & & & & $\mathrm{Ta}$ & 4 & & & \\
\hline o & 16 & 0.00136 & 0.24797 & 0.54574 & o & 65 & -0.02293 & 0.50 & 0.25989 & $\mathrm{Ta}$ & 5 & 0.35972 & 0.24873 & 0.75297 \\
\hline o & 17 & & & & o & 66 & 0.48723 & 0.50 & 0.28 & $\mathrm{Ta}$ & 6 & 0.49992 & 0.26244 & 0.74707 \\
\hline o & 18 & & & & o & 67 & 0.14 & 0. & 0.2 & $\mathrm{Ta}$ & 7 & 0.64128 & 0.74699 & 0.25906 \\
\hline 0 & 19 & 0.35609 & 0.30603 & 0.49 & o & 68 & 5 & 0. & & $\mathrm{Ta}$ & 8 & & & 613 \\
\hline o & 20 & 867 & 0.248 & 0.51 & o & 9 & & & & 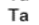 & 9 & & & \\
\hline o & 21 & & & & o & 70 & & & & a & 10 & & & \\
\hline o & 22 & & 0.7 & & o & 71 & & & & $\mathrm{Ta}$ & 13 & & & \\
\hline o & 23 & 0.431 & 0.79 & 0.28 & o & 72 & 0.02120 & 0.50 & 0.76 & Ta & 14 & 0.85371 & 0.00000 & -0.00122 \\
\hline o & 24 & & & & o & 73 & 0.12062 & 0. & & $\mathrm{Ta}$ & 15 & 0.00253 & 000 & \\
\hline o & 25 & 181 & & -0.0 & 0 & 74 & & & & $\mathrm{Ta}$ & 16 & & & \\
\hline o & 26 & -0.00 & & & 0 & 5 & & & & 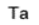 & 1 & & & \\
\hline & 27 & & & & o & 76 & 0 & & & a & 18 & & & 0.49816 \\
\hline o & 28 & & & & $\mathrm{Na}$ & 1 & 0.78 & & & $\mathrm{Ta}$ & 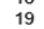 & & & -0.0 \\
\hline o & 29 & 376 & 0.794 & -0.00 & 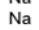 & 2 & & & & $\mathrm{Ta}$ & 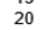 & & & \\
\hline o & 30 & 08 & 078 & & & 3 & 0.78 & & & $\mathrm{Ta}$ & 1 & 0.0 & 00 & -0 . \\
\hline o & 31 & & & & $\mathrm{Na}$ & 4 & & & & $\mathrm{Ta}$ & 2 & & & \\
\hline o & 32 & & & & & 5 & & & & $\mathrm{Ta}$ & 23 & & & 0.49172 \\
\hline 0 & 33 & & & & & 6 & & & & Ta & 24 & -0.00615 & 0.50000 & 0.50385 \\
\hline o & & & & & $\mathrm{N}$ & 7 & 0.78 & & & & & & & \\
\hline o & 35 & & & & $\mathrm{~N}$ & 8 & 0.21 & & & & & & & \\
\hline o & 36 & 0.001 & 0.752 & 0.545 & & 9 & & & & & & & & \\
\hline o & 37 & & & & & 10 & & & & & & & & \\
\hline & 38 & & & & & 11 & & & & & & & & \\
\hline & 35 & & & & $\mathrm{Na}$ & 12 & 0.71576 & 0.00000 & 0.48560 & & & & & \\
\hline 0 & & & & & & & & & & & & & & \\
\hline
\end{tabular}

Figure S16. Total density of state (DOS), some partial DOSs, the structural model of $\mathrm{Na}_{1.5} \mathrm{Ca}_{2} \mathrm{Ta}_{3} \mathrm{O}_{9.5} \mathrm{~N}_{0.5}$, and fractional coordinates of atoms in the calculated cell (The DFT 
calculation was performed using CASTEP code, exchange-correlation functional: GGA-PBE, pseudopotentials: ultrasoft, cutoff energy: $500 \mathrm{eV}$, k-point spacing: $\left.0.02 \AA^{-1}\right)$. The bandgap of the $\mathrm{Na}_{1.5} \mathrm{Ca}_{2} \mathrm{Ta}_{3} \mathrm{O}_{9.5} \mathrm{~N}_{0.5}$ was $1.36 \mathrm{eV}$. 




Figure S17. Bandgap of $\mathrm{Na}_{2} \mathrm{Ca}_{2} \mathrm{Ta}_{3} \mathrm{O}_{9} \mathrm{~N}$ with different $\mathrm{N}$-doped sites (calculation was performed using CASTEP code, exchange-correlation functional: GGA-PBE, pseudopotentials: ultrasoft, cutoff energy: $500 \mathrm{eV}$, k-point spacing: $\left.0.01 \AA^{-1}\right)$. The DFT calculation of the structure of $\mathrm{Na}_{2} \mathrm{Ca}_{2} \mathrm{Ta}_{3} \mathrm{O}_{9} \mathrm{~N}(\mathrm{O} / \mathrm{N}=9 / 1)$ indicated that replacement position of nitrogen is strongly dependent on the bandgap. When the $50 \% \mathrm{O}(1)$ sites are replaced with nitrogen, it become a metal (no bandgap). On the other hand, when the $50 \% \mathrm{O}(4)$ sites were replaced by nitrogen, the bandgap wa $0.55 \mathrm{eV}$. 


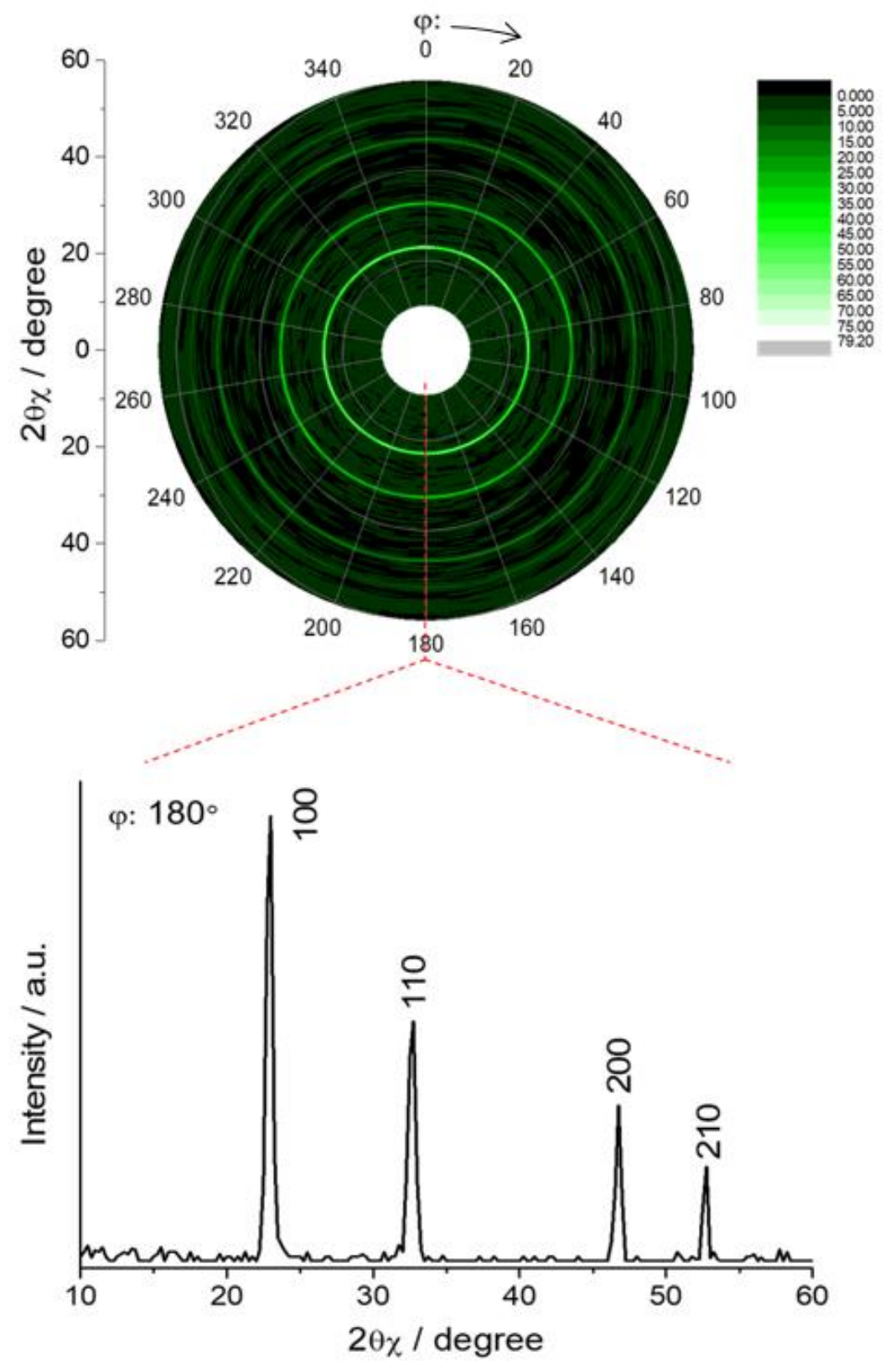

Figure S18. In-plane phi-scan XRD pattern of the monolayer $\mathrm{Ca}_{2} \mathrm{Ta}_{3} \mathrm{O}_{9.5} \mathrm{~N}_{0.5} 2 \mathrm{D}$ crystal film deposited on a Si substrate. The sample was rotated around the $\varphi$ axis from $0^{\circ}$ to $360^{\circ}$ in steps of $5^{\circ}$ and tilted step-by-step around the $2 \theta x$ axis from $10^{\circ}$ to $60^{\circ}$, in steps of $0.25^{\circ}$. 


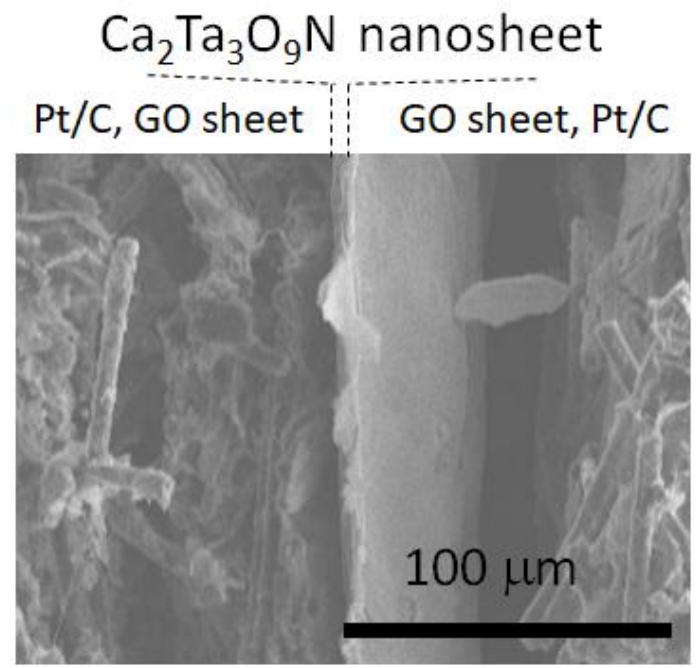

Figure S19. Cross sectional SEM image of hydrogen fuel cell using $\mathrm{Ca}_{2} \mathrm{Ta}_{3} \mathrm{O}_{9} \mathrm{~N}$ film as an electrolyte. 


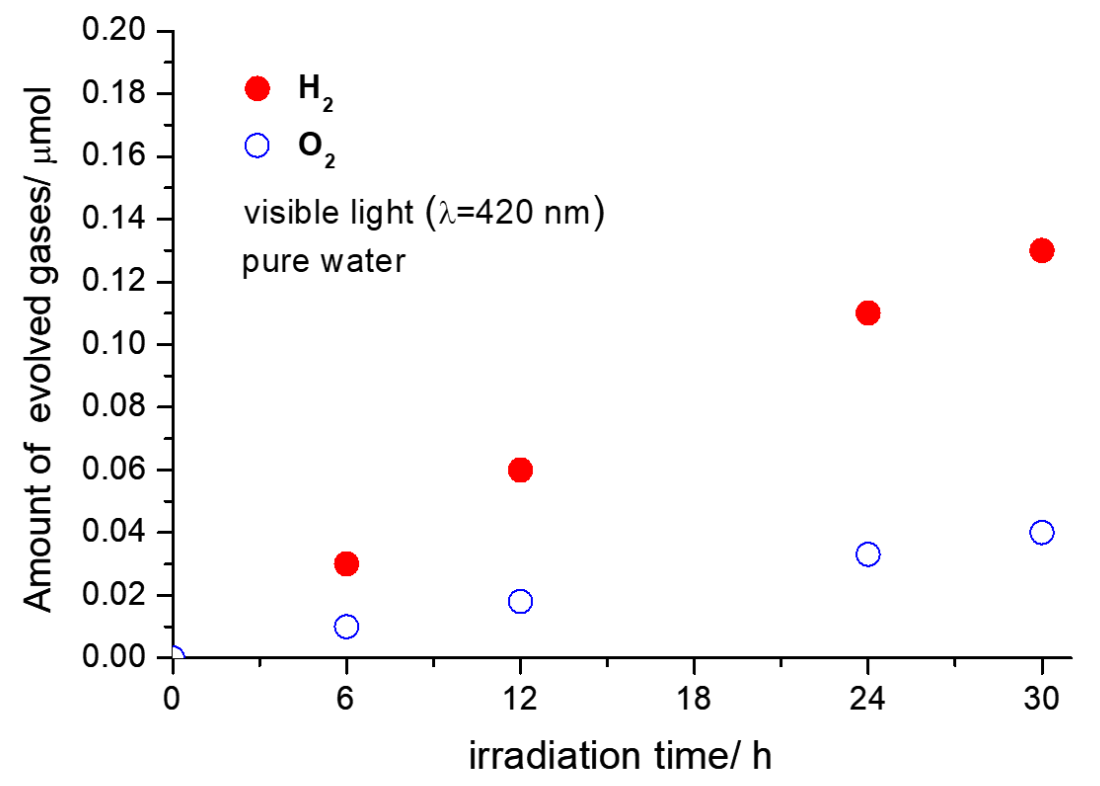

Figure S20. Time courses of hydrogen and oxygen generation over restacked 2D- $\mathrm{Ca}_{2} \mathrm{Ta}_{3} \mathrm{O}_{9} \mathrm{~N}$ (cocatalyst: $0.5 \mathrm{wt} \% \mathrm{RhCrO}_{x}$ and $0.5 \mathrm{wt} \% \mathrm{CoO}_{\mathrm{x}}$ ). Reaction conditions: catalyst, $20 \mathrm{mg}$ dissolved in $20 \mathrm{~mL}$ of distilled water; light source, LED visible light ( $\lambda=420$ $\mathrm{nm})$. 
Table S1. The light intensity and illumination area of monochromatic wavelength UV-light and LED light sources.

\begin{tabular}{ccc}
\hline Light source & Wavelength $(\mathrm{nm})$ & Intensity $\left(\mathrm{mW} / \mathrm{cm}^{2}\right)$ \\
\hline UV-light & $270 \mathrm{~nm}$ & $0.5 \mathrm{~mW}$ \\
$250-400 \mathrm{~nm}$ & $300 \mathrm{~nm}$ & $3.0 \mathrm{~mW}$ \\
$300 \mathrm{~W}$ & $320 \mathrm{~nm}$ & $3.0 \mathrm{~mW}$ \\
Xe lamp & $350 \mathrm{~nm}$ & $4.5 \mathrm{~mW}$ \\
\hline $420 \mathrm{~nm} \mathrm{LED}$ & $420 \mathrm{~nm}$ & $69 \mathrm{~mW}$ \\
$470 \mathrm{~nm}$ LED & $470 \mathrm{~nm}$ & $60 \mathrm{~mW}$ \\
\hline
\end{tabular}

